\title{
Eigenstates and Eigenvalues of Chain Hamiltonians Based on Multiparameter Braid Matrices for All Dimensions
}

\author{
B. Abdesselam ${ }^{1,2}$ and A. Chakrabarti ${ }^{3}$ \\ ${ }^{1}$ Laboratoire de Physique Théorique, Université d’Oran Es-Sénia, 31100 Oran, Algeria \\ ${ }^{2}$ Faculté des Sciences et de la Technologie, Centre Universitaire de Aïn Témouchent, 46000 Aïn Témouchent, Algeria \\ ${ }^{3}$ Centre de Physique Théorique, Ecole Polytechnique, 91128 Palaiseau Cedex, France
}

Correspondence should be addressed to A. Chakrabarti; chakra@cpht.polytechnique.fr

Received 4 September 2013; Accepted 14 October 2013

Academic Editors: U. Kulshreshtha and F. Sugino

Copyright ( 2013 B. Abdesselam and A. Chakrabarti. This is an open access article distributed under the Creative Commons Attribution License, which permits unrestricted use, distribution, and reproduction in any medium, provided the original work is properly cited.

\begin{abstract}
We study chain Hamiltonians derived from a class of multidimensional, multiparameter braid matrices introduced and explored in a series of previous papers. The $N^{2} \times N^{2}$ braid matrices (for all $N$ ) have $(1 / 2) N^{2}$ free parameters for even $N$ and $(1 / 2)(N+1)^{2}-1$ for $N$ odd. We present systematic explicit constructions for eigenstates and eigenvalues of chain Hamiltonians for $N=2,3,4$ and all chain lengths $r$. We derive explicitly the constraints imposed on these states by periodic (circular) boundary conditions. Our results thus cover both open and closed chains. We then indicate how our formalism can be extended for all $(N, r)$. The dependence of the eigenvalues on the free parameters is displayed explicitly, showing how the energy levels and their differences vary in a particular simple way with these parameters. Some perspectives are discussed in conclusion.
\end{abstract}

\section{Introduction}

In a series of previous paper [1-4], we have formulated and studied a class of $N^{2} \times N^{2}$ braid matrices $(N \geq 2)$ with free parameters whose numbers increase as $N^{2}$. Chain Hamiltonians corresponding to these matrices were also presented. Here, we undertake systematic study of chain Hamiltonians derived from these braid matrices presenting iterative and explicit constructions of eigenstates and eigenvalues for all dimensions $N$ and for all orders (chain lengths) $r$.

We recapitulate briefly the constructions of the braid matrices $\widehat{R}(\theta)$ satisfying (in standard notations used in [1-4])

$$
\widehat{R}_{12}(\theta) \widehat{R}_{23}\left(\theta+\theta^{\prime}\right) \widehat{R}_{12}\left(\theta^{\prime}\right)=\widehat{R}_{23}\left(\theta^{\prime}\right) \widehat{R}_{12}\left(\theta+\theta^{\prime}\right) \widehat{R}_{23}(\theta) .
$$

Our class has a nested sequence of projectors $P_{i j}^{(\epsilon)}$ as a basis which are defined as follows. For $N$ even $(N=2 n$, $n \geq 1$ ),

$$
P_{i j}^{(\epsilon)}=\frac{1}{2}\{(i i) \otimes(j j)+(\bar{i} \bar{i}) \otimes(\bar{j} \bar{j})
$$

$$
+\epsilon[(\bar{i} \bar{i}) \otimes(\overline{j j})+(\overline{i i}) \otimes(\bar{j} j)]\},
$$

where $i, j \in\{1, \ldots, N / 2\}, \epsilon= \pm, \bar{i}=N+1-i, \bar{j}=N+1-j$. Interchanging $j \rightleftharpoons \bar{j}$ on the right, one obtains $P_{i \bar{j}}^{(\epsilon)}$ with

$$
P_{i j}^{(\epsilon)}=P_{\bar{i} \bar{j}}^{(\epsilon)}, \quad P_{i \bar{j}}^{(\epsilon)}=P_{\bar{i} j}^{(\epsilon)} .
$$

(We use the notation $(a b)$ for a matrix with only one nonzero element, unity, on row $a$ and column $b$.) On such an orthonormal complete basis of projectors, one defines (with domains defined below (2))

$$
\widehat{R}(\theta)=\sum_{\epsilon} \sum_{i, j} e^{m_{i j}^{(\epsilon)} \theta}\left(P_{i j}^{(\epsilon)}+P_{i j}^{(\epsilon)}\right) .
$$

The crucial constraints on the free parameters

$$
m_{i j}^{(\epsilon)}=m_{i \bar{j}}^{(\epsilon)}
$$


give an $N^{2} \times N^{2}$ braid matrix with

$$
\frac{1}{2} N^{2}=2 n^{2}
$$

free parameters. For $N$ odd $(N=2 n-1 ; n \geq 2)$, using our definitions below (2),

$$
\begin{gathered}
\bar{n}=N-n+1=n, \\
P_{i n}^{(\epsilon)}=P_{i \bar{n}}^{(\epsilon)}=\frac{1}{2}\{(i i)+(\bar{i} \bar{i})+\epsilon[(i \bar{i})+(\bar{i} i)]\} \otimes(n n), \\
P_{n i}^{(\epsilon)}=P_{\bar{n} i}^{(\epsilon)}=\frac{1}{2}(n n) \otimes\{(i i)+(\bar{i} \bar{i})+\epsilon[(i \bar{i})+(\bar{i} i)]\}, \\
P_{n n}=(n n) \otimes(n n) .
\end{gathered}
$$

The braid matrix is now

$$
\begin{aligned}
\widehat{R}(\theta)= & e^{m_{n n} \theta} P_{n n}+\sum_{i, \epsilon}\left(e^{m_{n i}^{(\epsilon)} \theta} P_{n i}^{(\epsilon)}+e^{m_{i n}^{(\epsilon)} \theta} P_{i n}^{(\epsilon)}\right) \\
& +\sum_{i, j \epsilon} e^{m_{i j}^{(\epsilon)} \theta}\left(P_{i j}^{(\epsilon)}+P_{i \bar{j}}^{(\epsilon)}\right)
\end{aligned}
$$

$(i, j \in\{1, \ldots, n-1\}, \bar{i}, \bar{j} \in\{2 n-1, \ldots, n+1\}, \epsilon= \pm)$. The number of free parameters is now

$$
2 n^{2}-1=\frac{1}{2}(N+1)^{2}-1 .
$$

(An overall factor $e^{m_{n n} \theta}$ and redefinitions of the $m^{\prime} s, m_{n i}^{(\epsilon)}+$ $m_{n n} \rightarrow m_{n i}^{(\epsilon)}$, etc. convert our previous convention for odd $N$ to the present one, which is more convenient for what follows.)

Defining

$$
\dot{\widehat{R}}(0) \equiv\left(\frac{d}{d \theta} \widehat{R}(\theta)\right)_{\theta=0},
$$

the Hamiltonian acting on a chain of $r+1$ sites $(r \geq 1)$ is given by the standard

$$
H=\sum_{k=1}^{r} I \otimes \cdots \otimes I \otimes \dot{\widehat{R}}_{k, k+1}(0) \otimes I \otimes \cdots \otimes I,
$$

where $\dot{\widehat{R}}_{k, k+1}(0)$ acts on the sites $(k, k+1)$. For circular boundary conditions (or periodic),

$$
k+1=r+1 \approx 1 \text {. }
$$

The Hamiltonians inherit the free parameters (see (6), (9)) of $\widehat{R}$. This is the most striking features of our construction. The eigenvalues will be seen to depend linearly on these parameters (given by simple sums of the $m$ 's). So, by varying them, one can vary the differences between the energy levels. We will first present, in the following sections, explicit results for the simplest cases. Then, an iterative formalism will be implemented to generalize them. Also, we will start with open chains and subsequently impose the constraints (12) for periodicity.
2. $N=2 n=2$

The $4 \times 4$ braid matrix [3] is

$$
\widehat{R}(\theta)=\left|\begin{array}{cccc}
a_{+} & 0 & 0 & a_{-} \\
0 & a_{+} & a_{-} & 0 \\
0 & a_{-} & a_{+} & 0 \\
a_{-} & 0 & 0 & a_{+}
\end{array}\right|,
$$

where

$$
a_{ \pm}=\frac{1}{2}\left(e^{m_{11}^{(+)} \theta} \pm e^{m_{11}^{(-)} \theta}\right)
$$

Hence,

$$
\dot{\widehat{R}}(0)=\left|\begin{array}{cccc}
\mathrm{x}_{+} & 0 & 0 & \mathrm{x}_{-} \\
0 & \mathrm{x}_{+} & \mathrm{x}_{-} & 0 \\
0 & \mathrm{x}_{-} & \mathrm{x}_{+} & 0 \\
\mathrm{x}_{-} & 0 & 0 & \mathrm{x}_{+}
\end{array}\right|,
$$

where

$$
\mathrm{x}_{ \pm}=\frac{1}{2}\left(m_{11}^{(+)} \pm m_{11}^{(-)}\right)
$$

The chains Hamiltonian for $r+1$ sites are

$$
H^{(r)}=\dot{\widehat{\hat{R}}}_{12}(0)+\dot{\widehat{R}}_{23}(0)+\cdots+\dot{\hat{R}}_{r, r+1}(0)
$$

(adapting the notation of (11)). For circular boundary conditions (CBC),

$$
\dot{\widehat{R}}_{r, r+1}(0) \approx \dot{\widehat{R}}_{r, 1}(0) .
$$

The base spaces (for different $r$ 's) will be spanned by the tensor products of the fundamental states vectors

$$
|1\rangle \equiv\left|\begin{array}{l}
1 \\
0
\end{array}\right\rangle, \quad|\overline{1}\rangle \equiv\left|\begin{array}{l}
0 \\
1
\end{array}\right\rangle .
$$

Using the notations of our previous papers,

$$
|1 \overline{1} 1 \overline{1} 11\rangle \equiv|1\rangle \otimes|\overline{1}\rangle \otimes|1\rangle \otimes|\overline{1}\rangle \otimes|1\rangle \otimes|1\rangle
$$

and so on.

We now increase $r$ stepwise as follows.

2.1. $r=1$. Here,

$$
H=H^{(1)}=\dot{\widehat{R}}_{12}(0) .
$$

From (15), (19), and (20) (and with $\epsilon= \pm$ ),

$$
\begin{aligned}
& H^{(1)}(|11\rangle+\epsilon|\overline{11}\rangle)=m_{11}^{(\epsilon)}(|11\rangle+\epsilon|\overline{11}\rangle), \\
& H^{(1)}(|1 \overline{1}\rangle+\epsilon|\overline{1} 1\rangle)=m_{11}^{(\epsilon)}(|1 \overline{1}\rangle+\epsilon|\overline{1} 1\rangle) .
\end{aligned}
$$

The key to generalization $(r>1)$ is that, for the same $\epsilon$, these two eigenstates have the same eigenvalue. This degeneracy opens the road to a simple iteration. 
2.2. $r=2$. Now,

$$
H=H^{(2)}=\dot{\widehat{R}}_{12}(0)+\dot{\widehat{R}}_{23}(0) .
$$

Define

$$
\begin{aligned}
V_{\left(\epsilon_{1}, \epsilon_{2}, \epsilon_{3}\right)}= & \left(|1\rangle+\epsilon_{1}|\overline{1}\rangle\right)\left(|1\rangle+\epsilon_{2}|\overline{1}\rangle\right)\left(|1\rangle+\epsilon_{3}|\overline{1}\rangle\right) \\
= & \left(\left(|11\rangle+\epsilon_{1} \epsilon_{2}|\overline{11}\rangle\right)+\epsilon_{2}\left(|1 \overline{1}\rangle+\epsilon_{1} \epsilon_{2}|\overline{1} 1\rangle\right)\right) \\
& \times\left(|1\rangle+\epsilon_{3}|\overline{1}\rangle\right) \\
= & \left(|1\rangle+\epsilon_{1}|\overline{1}\rangle\right)\left(\left(|11\rangle+\epsilon_{2} \epsilon_{3}|\overline{11}\rangle\right)\right. \\
& \left.\quad+\epsilon_{3}\left(|1 \overline{1}\rangle+\epsilon_{2} \epsilon_{3}|\overline{1} 1\rangle\right)\right) .
\end{aligned}
$$

In (23), $\dot{\widehat{R}}_{12}(0)$ acts on the first two factors of $V$ and $\dot{\widehat{R}}_{23}(0)$ acts on the last two. Hence, form (22) and (25),

$$
\dot{\widehat{R}}_{12}(0) V_{\left(\epsilon_{1}, \epsilon_{2}, \epsilon_{3}\right)}=m_{11}^{\left(\epsilon_{1} \epsilon_{2}\right)} V_{\left(\epsilon_{1}, \epsilon_{2}, \epsilon_{3}\right)}
$$

and from (26)

$$
\dot{\widehat{R}}_{23}(0) V_{\left(\epsilon_{1}, \epsilon_{2}, \epsilon_{3}\right)}=m_{11}^{\left(\epsilon_{2} \epsilon_{3}\right)} V_{\left(\epsilon_{1}, \epsilon_{2}, \epsilon_{3}\right)} .
$$

Summing

$$
H^{(2)} V_{\left(\epsilon_{1}, \epsilon_{2}, \epsilon_{3}\right)}=\left(m_{11}^{\left(\epsilon_{1} \epsilon_{2}\right)}+m_{11}^{\left(\epsilon_{2} \epsilon_{3}\right)}\right) V_{\left(\epsilon_{1}, \epsilon_{2}, \epsilon_{3}\right)} .
$$

Since each $\left(\epsilon_{1}, \epsilon_{2}, \epsilon_{3}\right)$ can have values $( \pm)$ independently, we obtain a complete basis (for $r=2$ ) of eigenstates with the spectrum of eigenvalues

$$
\left(2 m_{11}^{(+)}, m_{11}^{(+)}+m_{11}^{(-)}, 2 m_{11}^{(-)}\right)
$$

with respective degeneracies (for the base space of 8 dimensions)

$$
2(1,2,1)
$$

The generalization is now evident.

2.3. General Case $r \geq 2$. Iterating factors as in (24) one can define

$$
\begin{aligned}
& V_{\left(\epsilon_{1}, \epsilon_{2}, \ldots, \epsilon_{r+1}\right)} \\
& \quad=\left(|1\rangle+\epsilon_{1}|\overline{1}\rangle\right)\left(|1\rangle+\epsilon_{2}|\overline{1}\rangle\right) \cdots\left(|1\rangle+\epsilon_{r+1}|\overline{1}\rangle\right) .
\end{aligned}
$$

Now, $H=H^{(r)}=\dot{\widehat{R}}_{12}(0)+\dot{\widehat{R}}_{23}(0)+\cdots+\dot{\widehat{R}}_{r, r+1}(0)$. In this sum, $\dot{\hat{R}}_{p, p+1}(0)$ acts on the factors

$$
\begin{aligned}
& \left(|1\rangle+\epsilon_{p}|\overline{1}\rangle\right)\left(|1\rangle+\epsilon_{p+1}|\overline{1}\rangle\right) \\
& =\left(\left(|11\rangle+\epsilon_{p} \epsilon_{p+1}|\overline{11}\rangle\right)+\epsilon_{p+1}\left(|1 \overline{1}\rangle+\epsilon_{p} \epsilon_{p+1}|\overline{1} 1\rangle\right)\right)
\end{aligned}
$$

giving

$$
\dot{\hat{R}}_{p, p+1}(0) V_{\left(\epsilon_{1}, \epsilon_{2}, \ldots, \epsilon_{r+1}\right)}=m_{11}^{\left(\epsilon_{p} \epsilon_{p+1}\right)} V_{\left(\epsilon_{1}, \epsilon_{2}, \ldots, \epsilon_{r+1}\right)}, \quad p=1, \ldots, r .
$$

Summing

$$
\begin{aligned}
& H^{(r)} V_{\left(\epsilon_{1}, \epsilon_{2}, \ldots, \epsilon_{r+1}\right)} \\
& =\left(m_{11}^{\left(\epsilon_{1} \epsilon_{2}\right)}+m_{11}^{\left(\epsilon_{2} \epsilon_{3}\right)}+\cdots+m_{11}^{\left(\epsilon_{r} \epsilon_{r+1}\right)}\right) V_{\left(\epsilon_{1}, \epsilon_{2}, \ldots, \epsilon_{r+1}\right)},
\end{aligned}
$$

the full spectrum is given by

$$
\left((r-k) m_{11}^{(+)}+k m_{11}^{(-)}\right), \quad(k=0,1,2, \ldots, r) .
$$

The respective multiplicities are given by the coefficients of the expansion

$$
2(x+y)^{r}=2 \sum_{k=0}^{r}\left(\begin{array}{l}
r \\
k
\end{array}\right) x^{r-k} y^{k} .
$$

This provides the complete basis of eigenstates spanning the base space of dimension $2^{r+1}$. Thus, we arrive (by iterating factors as in (32) at the complete solution for a chain of arbitrary length $((r+1)$ sites for any $r)$. We have obtained explicitly all eigenstates and the respective eigenvalues.

2.4. Cyclic Boundary Conditions (CBC). So far, we have been dealing with open chains. Now, we impose periodic (cyclic) boundary conditions, namely, (18). We start again with the simplest cases which again indicate the general solution.

2.4.1. $r=2$. From (24),

$$
\begin{aligned}
& V_{\left(\epsilon_{1}, \epsilon_{2}, \epsilon_{3}\right)} \\
& =\left(|1\rangle\left(|1\rangle+\epsilon_{2}|\overline{1}\rangle\right)|1\rangle+\epsilon_{1} \epsilon_{3}|\overline{1}\rangle\left(|1\rangle+\epsilon_{2}|\overline{1}\rangle\right)|\overline{1}\rangle\right)_{(\mathrm{P})} \\
& +\left(\epsilon_{3}|1\rangle\left(|1\rangle+\epsilon_{2}|\overline{1}\rangle\right)|\overline{1}\rangle\right. \\
& \left.\quad+\epsilon_{1}|\overline{1}\rangle\left(|1\rangle+\epsilon_{2}|\overline{1}\rangle\right)|1\rangle\right)_{(\mathrm{NP})^{\circ}}
\end{aligned}
$$

The part (P) satisfies the constraint (12) and the part (NP) does not. The part (NP) is eliminated in the following combination:

$$
\begin{aligned}
& \frac{1}{2}\left(V_{\left(+, \epsilon_{2},-\right)}+V_{\left(-, \epsilon_{2},+\right)}\right) \\
& \quad=|1\rangle\left(|1\rangle+\epsilon_{2}|\overline{1}\rangle\right)|1\rangle-|\overline{1}\rangle\left(|1\rangle+\epsilon_{2}|\overline{1}\rangle\right)|\overline{1}\rangle \\
& \quad=(|111\rangle-|\overline{1} 1 \overline{1}\rangle)+\epsilon_{2}(|1 \overline{1} 1\rangle-|\overline{111}\rangle) .
\end{aligned}
$$

The condition

$$
\epsilon_{1} \epsilon_{3}=-1
$$

and the sum imposed above leave only two eigenstates with the common eigenvalue

$$
\left(m_{11}^{(+)}+m_{11}^{(-)}\right) \text {. }
$$


It will be shown below that for all $r$ the extreme eigenvalues $\left(r m_{11}^{(+)}, r m_{11}^{(-)}\right)$are eliminated under $(\mathrm{CBC})$ and the dimension of the base space is divided by a factor 4 (for $N=2$ ).

2.4.2. General Case (All r). From (32), the (P) and (NP) parts are

$$
\begin{aligned}
& V_{\left(\epsilon_{1}, \epsilon_{2}, \ldots, \epsilon_{r}, \epsilon_{r+1}\right)} \\
& =\left(|1\rangle\left(|1\rangle+\epsilon_{2}|\overline{1}\rangle\right) \cdots\left(|1\rangle+\epsilon_{r}|\overline{1}\rangle\right)|1\rangle\right. \\
& \left.\quad+\epsilon_{1} \epsilon_{r+1}|\overline{1}\rangle\left(|1\rangle+\epsilon_{2}|\overline{1}\rangle\right) \cdots\left(|1\rangle+\epsilon_{r}|\overline{1}\rangle\right)|\overline{1}\rangle\right)_{(\mathrm{P})} \\
& +\left(\epsilon_{r+1}|1\rangle\left(|1\rangle+\epsilon_{2}|\overline{1}\rangle\right) \cdots\left(|1\rangle+\epsilon_{r}|\overline{1}\rangle\right)|\overline{1}\rangle\right. \\
& \left.\quad+\epsilon_{1}|\overline{1}\rangle\left(|1\rangle+\epsilon_{2}|\overline{1}\rangle\right) \cdots\left(|1\rangle+\epsilon_{r}|\overline{1}\rangle\right)|1\rangle\right)_{(\mathrm{NP})} .
\end{aligned}
$$

Thus, the direct generalization of (39) is

$$
\begin{aligned}
\frac{1}{2} & \left(V_{\left(+, \epsilon_{2}, \ldots, \epsilon_{r},-\right)}+V_{\left(-, \epsilon_{2}, \ldots, \epsilon_{r},+\right)}\right) \\
\quad= & |1\rangle\left(|1\rangle+\epsilon_{2}|\overline{1}\rangle\right) \cdots\left(|1\rangle+\epsilon_{r}|\overline{1}\rangle\right)|1\rangle \\
& -|\overline{1}\rangle\left(|1\rangle+\epsilon_{2}|\overline{1}\rangle\right) \cdots\left(|1\rangle+\epsilon_{r}|\overline{1}\rangle\right)|\overline{1}\rangle,
\end{aligned}
$$

which provides the $2^{r-1}$ eigenstates satisfying $(\mathrm{CBC})$ with the spectrum of eigenvalues

$$
\left((r-k) m_{11}^{(+)}+k m_{11}^{(-)}\right), \quad(k=1,2, \ldots, r-1) .
$$

\section{3. $N=4$}

We continue to study even dimensions. For $N=2$, we had to deal with one pair of indices $(|1\rangle,|\overline{1}\rangle$ of Section 2). For $N=2 n$, the states involve tensor products of $(|1\rangle,|\overline{1}\rangle ;|2\rangle,|\overline{2}\rangle ; \ldots ;|n\rangle,|\bar{n}\rangle)$. For $n=2$, the basis involving $(|1\rangle,|\overline{1}\rangle ;|2\rangle,|\overline{2}\rangle)$ will be studied using notations that facilitate the passage to all $n$. For states involving only $(|1\rangle,|\overline{1}\rangle)$ or $(|2\rangle,|\overline{2}\rangle)$, the results will be entirely analogous to those of Section $2(n=1)$. But now, one has to include states involving both pairs. Changing the notations of [3] for convenience, we write (for $N=4$ ) the $16 \times 16$ Hamiltonian as

$$
\dot{\hat{R}}(0)=\left|\begin{array}{cccc}
D_{11} & 0 & 0 & A_{1 \overline{1}} \\
0 & D_{22} & A_{2 \overline{2}} & 0 \\
0 & A_{\overline{2} 2} & D_{\overline{22}} & 0 \\
A_{\overline{1} 1} & 0 & 0 & D_{\overline{1} \overline{1}}
\end{array}\right|,
$$

where

$$
D_{11}=D_{\overline{1} \overline{1}}=\left(\begin{array}{cccc}
a_{+} & 0 & 0 & 0 \\
0 & b_{+} & 0 & 0 \\
0 & 0 & b_{+} & 0 \\
0 & 0 & 0 & a_{+}
\end{array}\right),
$$

$$
D_{22}=D_{\overline{2} \overline{2}}=\left(\begin{array}{cccc}
c_{+} & 0 & 0 & 0 \\
0 & d_{+} & 0 & 0 \\
0 & 0 & d_{+} & 0 \\
0 & 0 & 0 & c_{+}
\end{array}\right) \text {, }
$$$$
A_{1 \overline{1}}=A_{\overline{1} 1}=\left(\begin{array}{cccc}
0 & 0 & 0 & a_{-} \\
0 & 0 & b_{-} & 0 \\
0 & b_{-} & 0 & 0 \\
a_{-} & 0 & 0 & 0
\end{array}\right) \text {, }
$$$$
A_{2 \overline{2}}=A_{\overline{2} 2}=\left(\begin{array}{cccc}
0 & 0 & 0 & c_{-} \\
0 & 0 & d_{-} & 0 \\
0 & d_{-} & 0 & 0 \\
c_{-} & 0 & 0 & 0
\end{array}\right),
$$

$$
\begin{array}{ll}
a_{ \pm}=\frac{1}{2}\left(m_{11}^{(+)} \pm m_{11}^{(-)}\right), & b_{ \pm}=\frac{1}{2}\left(m_{12}^{(+)} \pm m_{12}^{(-)}\right), \\
c_{ \pm}=\frac{1}{2}\left(m_{21}^{(+)} \pm m_{21}^{(-)}\right), & d_{ \pm}=\frac{1}{2}\left(m_{22}^{(+)} \pm m_{22}^{(-)}\right) .
\end{array}
$$

As compared to $m_{11}^{( \pm)}$of Section 2, there are now 8 independent parameters.

The fundamental basis states are (as compared to (19))

$$
\begin{aligned}
& \left.|1\rangle \equiv\left|\begin{array}{l}
1 \\
0 \\
0 \\
0
\end{array}\right|, \quad|\overline{1}\rangle \equiv \mid \begin{array}{l}
0 \\
0 \\
0 \\
1
\end{array}\right), \\
& |2\rangle \equiv\left|\begin{array}{l}
0 \\
1 \\
0 \\
0
\end{array}\right\rangle, \quad|\overline{2}\rangle \equiv\left|\begin{array}{l}
0 \\
0 \\
1 \\
0
\end{array}\right\rangle \text {. }
\end{aligned}
$$

The tensor products, using our standard notations, are denoted as

$$
|i j\rangle=|i\rangle \otimes|j\rangle,
$$

where, independently, $i=(1, \overline{1}, 2, \overline{2}), j=(1, \overline{1}, 2, \overline{2})$, and correspondingly, $\bar{i}=(\overline{1}, 1, \overline{2}, 2)$ and similarly for $j$.

The crucial constraint (always to be kept in mind)

$$
m_{i j}^{(\epsilon)}=m_{i j}^{(\epsilon)}=m_{i j}^{(\epsilon)}=m_{i j}^{(\epsilon)}
$$

is incorporated in the blocks of (45) through (45), (47). We will write $m_{12}^{(\epsilon)}$ for $\left(m_{12}^{(\epsilon)}, m_{\overline{12}}^{(\epsilon)}, m_{12}^{(\epsilon)}, m_{12}^{(\epsilon)}\right)$ and so on. A fundamental consequence is

$$
\dot{\hat{R}}(0)|i j\rangle=\frac{1}{2}\left(m_{i j}^{(+)}+m_{i j}^{(-)}\right)|i j\rangle+\frac{1}{2}\left(m_{i j}^{(+)}-m_{i j}^{(-)}\right)|\overline{i j}\rangle
$$


for

$$
i=(1, \overline{1}, 2, \overline{2}) \quad \text { with } \bar{i}=(\overline{1}, 1, \overline{2}, 2)
$$

and similarly for $j$. Hence,

$$
\dot{\hat{R}}(0)(|i j\rangle+\epsilon|\overline{i j}\rangle)=m_{i j}^{(\epsilon)}(|i j\rangle+\epsilon|\overline{i j}\rangle),
$$

where $\epsilon= \pm$ and $(i, j)$ assume the values $(1, \overline{1}, 2, \overline{2})$ independently. Hence, due to (50), also

$$
\dot{\hat{R}}(0)(|i \bar{j}\rangle+\epsilon|\bar{i} j\rangle)=m_{i j}^{(\epsilon)}(|i \bar{j}\rangle+\epsilon|\bar{i} j\rangle) .
$$

3.1. $(r=2)$. Define

$$
V_{\left(\epsilon_{1}, \epsilon_{2}, \epsilon_{3}\right)}=\left(\left|i_{1}\right\rangle+\epsilon_{1}\left|\bar{i}_{1}\right\rangle\right)\left(\left|i_{2}\right\rangle+\epsilon_{2}\left|\bar{i}_{2}\right\rangle\right)\left(\left|i_{3}\right\rangle+\epsilon_{3}\left|\bar{i}_{3}\right\rangle\right)
$$

exactly as in (25), (26) but with the domain $(1,2)$ for each index $\left(i_{1}, i_{2}, i_{3}\right)$. (Changing any $i$ to $\bar{i}$ amounts to the same $V$ with the corresponding $\epsilon$ as factor.) Consider

$$
\begin{aligned}
& V_{\left(\epsilon_{1}, \epsilon_{2}, \epsilon_{3}\right)} \\
& =\left(\left(\left|i_{1} i_{2}\right\rangle+\epsilon_{1} \epsilon_{2}\left|\bar{i}_{1} \bar{i}_{2}\right\rangle\right)+\epsilon_{2}\left(\left|i_{1} \bar{i}_{2}\right\rangle+\epsilon_{1} \epsilon_{2}\left|\bar{i}_{1} i_{2}\right\rangle\right)\right) \\
& \quad \times\left(\left|i_{3}\right\rangle+\epsilon_{3}\left|\bar{i}_{3}\right\rangle\right) \\
& =\left(\left|i_{1}\right\rangle+\epsilon_{1}\left|\bar{i}_{1}\right\rangle\right) \\
& \quad \times\left(\left(\left|i_{2} i_{3}\right\rangle+\epsilon_{2} \epsilon_{3}\left|\bar{i}_{2} \bar{i}_{3}\right\rangle\right)+\epsilon_{3}\left(\left|i_{2} \bar{i}_{3}\right\rangle+\epsilon_{2} \epsilon_{3}\left|\bar{i}_{2} i_{3}\right\rangle\right)\right) .
\end{aligned}
$$

Hence, (analogously to (27)-(29))

$$
\begin{aligned}
H^{(2)} V_{\left(\epsilon_{1}, \epsilon_{2}, \epsilon_{3}\right)} & =\left(\dot{\widehat{R}}_{12}(0)+\dot{\widehat{R}}_{23}(0)\right) V_{\left(\epsilon_{1}, \epsilon_{2}, \epsilon_{3}\right)} \\
& =\left(m_{i_{1} i_{2}}^{\left(\epsilon_{1} \epsilon_{2}\right)}+m_{i_{2} i_{3}}^{\left(\epsilon_{2} \epsilon_{3}\right)}\right) V_{\left(\epsilon_{1}, \epsilon_{2}, \epsilon_{3}\right)} .
\end{aligned}
$$

The difference from (29) is that here the eigenvalues (taking (50) into account) have the domain

$$
\begin{array}{ll}
\left(m_{11}^{\left(\epsilon_{1} \epsilon_{2}\right)}+m_{11}^{\left(\epsilon_{2} \epsilon_{3}\right)}\right), & \left(m_{12}^{\left(\epsilon_{1} \epsilon_{2}\right)}+m_{12}^{\left(\epsilon_{2} \epsilon_{3}\right)}\right), \\
\left(m_{12}^{\left(\epsilon_{1} \epsilon_{2}\right)}+m_{21}^{\left(\epsilon_{2} \epsilon_{3}\right)}\right), & \left(m_{12}^{\left(\epsilon_{1} \epsilon_{2}\right)}+m_{22}^{\left(\epsilon_{2} \epsilon_{3}\right)}\right), \\
\left(m_{21}^{\left(\epsilon_{1} \epsilon_{2}\right)}+m_{21}^{\left(\epsilon_{2} \epsilon_{3}\right)}\right), & \left(m_{21}^{\left(\epsilon_{1} \epsilon_{2}\right)}+m_{12}^{\left(\epsilon_{2} \epsilon_{3}\right)}\right), \\
\left(m_{22}^{\left(\epsilon_{1} \epsilon_{2}\right)}+m_{21}^{\left(\epsilon_{2} \epsilon_{3}\right)}\right), & \left(m_{22}^{\left(\epsilon_{1} \epsilon_{2}\right)}+m_{22}^{\left(\epsilon_{2} \epsilon_{3}\right)}\right) .
\end{array}
$$

For each case, independently,

$$
\left(\epsilon_{1}, \epsilon_{2}\right)=(+,-), \quad\left(\epsilon_{2}, \epsilon_{3}\right)=(+,-) .
$$

Each entry in (57) provides 8 possibilities (including multiplicities, easily counted) and thus in total spans the $4^{2+1}=$ $8 \times 8=64$ dimensional base space for $r=2$.
3.2. General Case $(r=2,3,4, \ldots)$. We generalized (24) to (55) in passing from $N=2$ to $N=4$. Similarly, for all $r$, we generalize (32) to

$$
\begin{aligned}
& V_{\left(\epsilon_{1}, \epsilon_{2}, \ldots, \epsilon_{r+1}\right)} \\
& =\left(\left|i_{1}\right\rangle+\epsilon_{1}\left|\bar{i}_{1}\right\rangle\right)\left(\left|i_{2}\right\rangle+\epsilon_{2}\left|\bar{i}_{2}\right\rangle\right) \cdots\left(\left|i_{r+1}\right\rangle+\epsilon_{r+1}\left|\bar{i}_{r+1}\right\rangle\right)
\end{aligned}
$$

with the domain $(1,2)$ for each $i$. Now, $H^{(r)}=\dot{\widehat{R}}_{12}(0)+\dot{\widehat{R}}_{23}(0)+$ $\cdots+\dot{\widehat{R}}_{r, r+1}(0)$ and the evident generalization of (35) is

$$
\begin{aligned}
& H^{(r)} V_{\left(\epsilon_{1}, \epsilon_{2}, \ldots, \epsilon_{r+1}\right)} \\
& \quad=\left(m_{i_{1} i_{2}}^{\left(\epsilon_{1} \epsilon_{2}\right)}+m_{i_{2} i_{3}}^{\left(\epsilon_{2} \epsilon_{3}\right)}+\cdots+m_{i_{r} i_{r+1}}^{\left(\epsilon_{r} \epsilon_{r+1}\right)}\right) V_{\left(\epsilon_{1}, \epsilon_{2}, \ldots, \epsilon_{r+1}\right)} .
\end{aligned}
$$

Each term can contribute

$$
\left(m_{11}^{( \pm)}, m_{12}^{( \pm)}, m_{21}^{( \pm)}, m_{22}^{( \pm)}\right)
$$

for the full range of the $i$ 's and the $\epsilon$ 's. But for a given value of $i_{2}$ in $m_{i_{1} i_{2}}^{\left(\epsilon_{1} \epsilon_{2}\right)}$, one must have the same $i_{2}$ in $m_{i_{2} i_{3}}^{\left(\epsilon_{2} \epsilon_{3}\right)}$ and so on. This introduces constraints. Thus, for $r=3(r+1=4)$, for fixed $i$ 's, the $\epsilon$ 's provide $2^{4}$ possibilities (including multiplicities) and for each set of $\epsilon$ 's, the $i$ 's (each being 1 or 2 ) furnish $2^{4}$ possibilities. These together indeed span the base space of $2^{4} \times 2^{4}=4^{3+1}$ dimensions.

3.3. $C B C$. Circular boundary conditions can be imposed now. As compared to the case $N=2$, along with strong analogies, there are crucial differences. One evident difference is the larger domains of the indices. The constraints $i_{r+1}=i_{1}$ leave, for $N=2$, the possibilities

$$
i_{r+1}=i_{1}=(1, \overline{1}) .
$$

Now, one can have

$$
i_{r+1}=i_{1}=(1, \overline{1}, 2, \overline{2}) \text {. }
$$

For $N=2 n$, one can have

$$
i_{r+1}=i_{1}=(1, \overline{1}, 2, \overline{2}, \ldots, n, \bar{n}) .
$$

But there is a somewhat more subtle difference. The fundamental constraint (5) and the symmetries of the projectors (see (3)) lead to

$$
m_{i j}^{(\epsilon)}=m_{i \bar{j}}^{(\epsilon)}=m_{\bar{i} j}^{(\epsilon)}=m_{i j}^{(\epsilon)}
$$

for our free parameters. But we do not indent to impose, diminishing number of free parameters, which are an essential feature of our formalism, additional restrictions as $m_{i j}^{(\epsilon)}=$ $m_{j i}^{(\epsilon)}(i \neq j)$, and so on. Let us explore the consequences for (CBC) for $N>2$. 
3.3.1. $r=2$. From (53), (54), one obtains periodic states by starting with

$$
\begin{aligned}
& V_{\left(\epsilon_{1}, \epsilon_{2}\right)}^{(i, j, i)}=\left(|i j i\rangle+\epsilon_{1} \epsilon_{2}|\bar{i} j \bar{i}\rangle\right)+\left(\epsilon_{1}|\bar{i} j i\rangle+\epsilon_{2}|i \overline{j i}\rangle\right), \\
& V_{\left(\epsilon_{1}, \epsilon_{2}\right)}^{(i, j, \bar{i})}=\left(|i j \bar{j}\rangle+\epsilon_{1} \epsilon_{2}|\bar{i} j i\rangle\right)+\left(\epsilon_{1}|\bar{i} \bar{j}\rangle+\epsilon_{2}|i j i\rangle\right)
\end{aligned}
$$

as

$$
\begin{aligned}
& \frac{1}{2}\left(V_{(+,-)}^{(i, j, i)}+V_{(-,+)}^{(i, j, i)}\right)=(|i j i\rangle-|\bar{i} j \bar{i}\rangle) \\
& \frac{1}{2}\left(V_{(+,-)}^{(i, j, \bar{i})}-V_{(-,+)}^{(i, j, \bar{i})}\right)=(|\bar{i} j \bar{i}\rangle-|i j i\rangle) .
\end{aligned}
$$

But for $j=(i, \bar{i})$, these are not eigenstates of $H^{(2)}$ if $m_{12} \neq m_{21}$. For $j=(i, \bar{i})$ only, one obtains eigenvalues

$$
\left(m_{i i}^{(+)}+m_{i i}^{(-)}\right) \text {. }
$$

3.3.2. $r>2$. Displaying explicitly the indices in the generalizations of (69), (70), the periodic states are

$$
\begin{aligned}
& \frac{1}{2}\left(V_{\left(+, \epsilon_{2}, \epsilon_{3}, \ldots, \epsilon_{r-1},-\right)}^{\left(i_{1}, i_{2}, \ldots, i_{r}, i_{1}\right)}+V_{\left(-, \epsilon_{2}, \epsilon_{3}, \ldots, \epsilon_{r-1},+\right)}^{\left(i_{1}, i_{2}, \ldots, i_{r}, i_{1}\right)}\right) \\
& \frac{1}{2}\left(V_{\left(+, \epsilon_{2}, \epsilon_{3}, \ldots, \epsilon_{r-1},-\right)}^{\left(i_{1}, i_{2}, \ldots, i_{r}, \bar{i}_{1}\right)}-V_{\left(+, \epsilon_{2}, \epsilon_{3}, \ldots, \epsilon_{r-1},-\right)}^{\left(i_{1}, i_{2}, \ldots, i_{r}, \bar{i}_{1}\right)}\right) .
\end{aligned}
$$

They are eigenstates only under constraints

$$
i_{2}=\left(i_{1}, \bar{i}_{1}\right), \quad i_{r}=\left(i_{1}, \bar{i}_{1}\right)
$$

(independent of each other). The first and the second terms of (72) or (3.30) contribute to differing eigenvalues by

$$
\left(m_{i_{1} i_{2}}^{(+)}+m_{i_{r} i_{1}}^{(-)}\right)-\left(m_{i_{1} i_{2}}^{(-)}+m_{i_{r} i_{1}}^{(+)}\right)
$$

which vanishes for (73) (along with (66)). Hence the results. For $r=2$, there is one constraint less. The restrictions on the eigenvalues and on the dimensions of the base spaces can now be obtained in a straightforward fashion. Along with, as in Section 2, $\epsilon_{1} \epsilon_{r}=-1$, there are now (71) and (73).

We have preferred to study the case $N=4$ (rather than $N=3)$ directly after $N=2$, since all even $(N=2 n)$ cases can be treated coherently together while all odd ones $(N=$ $2 n-1, n=2,3, \ldots)$ have features in common which are absent for $N=2 n$. The prototype is provided by $N=3$. We study this case in some detail in the following section. Then, we will only try to understand generalizations necessary for arbitrary dimensions $N=(2,3,4,5,6, \ldots)$.

\section{4. $N=3$}

The definition below (2), $\bar{i}=N+1-i(\overline{\bar{i}}=N+1-\bar{i}=i)$, gives, for $N=2 n-1$,

$$
\bar{n}=N+1-n=n \text {. }
$$

While, for all other indices, $\bar{i} \neq i$. This is the central fact inducing special properties for odd $N$ (for even $N$ no $i=\bar{i}$ ).
We now study such features in some detail for $N=3$. The $9 \times 9$ braid matrices are (in notations analogous to (45))

$$
\widehat{R}(\theta)=\left|\begin{array}{ccc}
D_{11} & 0 & A_{\overline{1}} \\
0 & D_{22} & 0 \\
A_{\overline{1} 1} & 0 & D_{\overline{1} \overline{1}}
\end{array}\right|,
$$

where

$$
\begin{gathered}
D_{11}=D_{\overline{1} \overline{1}}=\left(\begin{array}{ccc}
a_{+} & 0 & 0 \\
0 & b_{+} & 0 \\
0 & 0 & a_{+}
\end{array}\right), \\
D_{22}=\left(\begin{array}{ccc}
c_{+} & 0 & c_{-} \\
0 & d & 0 \\
c_{-} & 0 & c_{+}
\end{array}\right), \\
A_{1 \overline{1}}=A_{\overline{1} 1}=\left(\begin{array}{ccc}
0 & 0 & a_{-} \\
0 & b_{-} & 0 \\
a_{-} & 0 & 0
\end{array}\right),
\end{gathered}
$$

where

$$
\begin{gathered}
a_{ \pm}=\frac{1}{2}\left(e^{m_{11}^{(+)} \theta} \pm e^{m_{11}^{(-)} \theta}\right), \quad b_{ \pm}=\frac{1}{2}\left(e^{m_{12}^{(+)} \theta} \pm e^{m_{12}^{(-)} \theta}\right), \\
c_{ \pm}=\frac{1}{2}\left(e^{m_{21}^{(+)} \theta} \pm e^{m_{21}^{(-)} \theta}\right), \quad d=e^{m_{22} \theta} .
\end{gathered}
$$

As compared to our conventions for odd $N$ in previous papers $[1,3,5]$, the above one differs by the introduction of an overall factor $e^{m_{22} \theta}$ and redefinitions (for $\left.(i, j) \neq(2,2)\right) m_{i j}^{( \pm)}+m_{22} \rightarrow$ $m_{i j}^{( \pm)}$. This is convenient for our present purposes. Now,

$$
\dot{\hat{R}}(0)=\left|\begin{array}{ccccccccc}
\alpha_{+} & 0 & 0 & 0 & 0 & 0 & 0 & 0 & \alpha_{-} \\
0 & \beta_{+} & 0 & 0 & 0 & 0 & 0 & \beta_{-} & 0 \\
0 & 0 & \alpha_{+} & 0 & 0 & 0 & \alpha_{-} & 0 & 0 \\
0 & 0 & 0 & \gamma_{+} & 0 & \gamma_{-} & 0 & 0 & 0 \\
0 & 0 & 0 & 0 & \gamma & 0 & 0 & 0 & 0 \\
0 & 0 & 0 & \gamma_{-} & 0 & \gamma_{+} & 0 & 0 & 0 \\
0 & 0 & \alpha_{-} & 0 & 0 & 0 & \alpha_{+} & 0 & 0 \\
0 & \beta_{-} & 0 & 0 & 0 & 0 & 0 & \beta_{+} & 0 \\
\alpha_{-} & 0 & 0 & 0 & 0 & 0 & 0 & 0 & \alpha_{+}
\end{array}\right|,
$$

where

$$
\begin{gathered}
\alpha_{ \pm}=\frac{1}{2}\left(m_{11}^{(+)} \pm m_{11}^{(-)}\right), \quad \beta_{ \pm}=\frac{1}{2}\left(m_{12}^{(+)} \pm m_{12}^{(-)}\right), \\
\gamma_{ \pm}=\frac{1}{2}\left(m_{21}^{(+)} \pm m_{21}^{(-)}\right), \quad \delta=m_{22} .
\end{gathered}
$$

(Compare this to (47), (48).) We have now 7 free parameters. The chain Hamiltonian is now ( $I$ being a $9 \times 9$ unit matrix)

$$
\begin{aligned}
H^{(r)} & =\sum_{k=1}^{r} I \otimes \cdots \otimes \dot{\hat{R}}(0)_{k, k+1} \otimes \cdots \otimes I \\
& \equiv \dot{\hat{R}}_{12}(0)+\dot{\widehat{R}}_{23}(0)+\cdots+\dot{\widehat{R}}_{r, r+1}(0) \quad(r=2,3, \ldots) .
\end{aligned}
$$

Again for periodicity for $k+1=r+1 \approx 1$, the generalizations for $N=(5,7, \ldots)$ are straight forward. 
4.1. Eigenstates and Eigenvalues. The base space is now spanned by tensor products of the fundamental states

$$
\left(\left|\begin{array}{l}
1 \\
0 \\
0
\end{array}\right\rangle,\left|\begin{array}{l}
0 \\
1 \\
0
\end{array}\right\rangle,\left|\begin{array}{l}
0 \\
0 \\
1
\end{array}\right\rangle,\right) \equiv(|1\rangle,|2\rangle,|\overline{1}\rangle) .
$$

The eigenstates and eigenvalues of $\dot{\widehat{R}}_{12}(0)\left(\equiv H^{(1)}\right)$ are (with $\epsilon= \pm$ )

$$
\begin{aligned}
H^{(1)}(|11\rangle+\epsilon|\overline{1} 1\rangle) & =m_{11}^{(\epsilon)}(|11\rangle+\epsilon|\overline{11}\rangle), \\
H^{(1)}(|1 \overline{1}\rangle+\epsilon|\overline{1} 1\rangle) & =m_{11}^{(\epsilon)}(|1 \overline{1}\rangle+\epsilon|\overline{1} 1\rangle), \\
H^{(1)}(|12\rangle+\epsilon|\overline{1} 2\rangle) & =m_{12}^{(\epsilon)}(|12\rangle+\epsilon|\overline{1} 2\rangle), \\
H^{(1)}(|21\rangle+\epsilon|2 \overline{1}\rangle) & =m_{21}^{(\epsilon)}(|21\rangle+\epsilon|2 \overline{1}\rangle), \\
H^{(1)}|22\rangle & =m_{22}|22\rangle .
\end{aligned}
$$

Comparing this with (53), one notices how the 16dimensional basis for $N=4$ is reduced ("contracted") to a 9-dimensional one for the identification ( for $N=3$ )

$$
2=\overline{2} \text {. }
$$

We will come back to this point.

4.1.1. $r=2$. We continue to elucidate the role of (84) by treating this case in detail. Here,

$$
H^{(2)}=\dot{\widehat{R}}_{12}(0)+\dot{\widehat{R}}_{23}(0) \text {. }
$$

For states involving only $(1, \overline{1})$, one proceeds exactly as for $N=2$ (see (24)-(32)). One thus recovers formally the eight eigenstates not involving $|2\rangle$. But now, one also has the following possibilities in the iterative structure of the eigenstates:

$$
\begin{array}{ll}
\text { (1) } & \left(|11\rangle+\epsilon_{1}|\overline{1} \overline{1}\rangle\right)|2\rangle+\epsilon_{2}\left(|1 \overline{1}\rangle+\epsilon_{1}|\overline{1} 1\rangle\right)|2\rangle \\
= & |1\rangle\left(|12\rangle+\epsilon_{2}|\overline{1} 2\rangle\right)+\epsilon_{1}|\overline{1}\rangle\left(|\overline{1} 2\rangle+\epsilon_{2}|12\rangle\right), \\
\text { (2) } \quad\left(|12\rangle+\epsilon_{1}|\overline{1} 2\rangle\right)\left(|1\rangle+\epsilon_{2}|\overline{1}\rangle\right) & \left(|1\rangle+\epsilon_{1}|\overline{1}\rangle\right)\left(|21\rangle+\epsilon_{2}|2 \overline{1}\rangle\right), \\
\text { (3) } \quad\left(|21\rangle+\epsilon_{1}|2 \overline{1}\rangle\right)\left(|1\rangle+\epsilon_{2}|\overline{1}\rangle\right) \\
=|2\rangle\left(\left(|11\rangle+\epsilon_{1} \epsilon_{2}|\overline{11}\rangle\right)+\epsilon_{2}\left(|1 \overline{1}\rangle+\epsilon_{1} \epsilon_{2}|\overline{1} 1\rangle\right)\right), \\
\text { (4) } \quad(|12\rangle+\epsilon|\overline{1} 2\rangle)|2\rangle=(|1\rangle+\epsilon|\overline{1}\rangle)|22\rangle, \\
\text { (5) }(|21\rangle+\epsilon|2 \overline{1}\rangle)|2\rangle=|2\rangle(|12\rangle+\epsilon|\overline{1} 1\rangle), \\
\text { (6) }|22\rangle(|1\rangle+\epsilon|\overline{1}\rangle)=|2\rangle(|21\rangle+\epsilon|2 \overline{1}\rangle), \\
\text { (7) }|222\rangle .
\end{array}
$$

The total action of $H^{(2)}$ is evident form the left and the right factorizations displayed. The eigenvalues are, respectively,

$$
\begin{aligned}
& \text { (1) }\left(m_{11}^{\left(\epsilon_{1}\right)}+m_{12}^{\left(\epsilon_{2}\right)}\right), \\
& \text { (2) }\left(m_{12}^{\left(\epsilon_{1}\right)}+m_{21}^{\left(\epsilon_{2}\right)}\right), \\
& \text { (3) }\left(m_{21}^{\left(\epsilon_{1}\right)}+m_{11}^{\left(\epsilon_{1} \epsilon_{2}\right)}\right), \\
& \text { (4) }\left(m_{12}^{(\epsilon)}+m_{22}\right), \\
& \text { (5) }\left(m_{21}^{(\epsilon)}+m_{12}^{(\epsilon)}\right), \\
& \text { (6) }\left(m_{22}+m_{21}^{(\epsilon)}\right), \\
& \text { (7) } 2 m_{22} .
\end{aligned}
$$

Counting the possible values of the $\epsilon$ 's, these provide $(12+6+$ $1)=19$ eigenstates. Along with 8 states involving only $(1, \overline{1})$, one thus obtains the full basis of 27 dimensions for $r=2$ (three sites with three possibilities $(1, \overline{1}, 2)$ for each). It is again instructive to note how the corresponding results for $N=4$ (54)-(56) reduce to those for $N=3$ under (84).

4.1.2. $r=3$. Before passing to the general case, it is helpful to display the role of the state $|2\rangle$ for $r=3$. Consider the state

$$
\left(|i\rangle+\epsilon_{1}|\bar{i}\rangle\right)\left(|j\rangle+\epsilon_{2}|\bar{j}\rangle\right)\left(|k\rangle+\epsilon_{3}|\bar{k}\rangle\right)\left(|l\rangle+\epsilon_{4}|\bar{l}\rangle\right) .
$$

Now, $H^{(3)}=\dot{\widehat{R}}_{12}(0)+\dot{\widehat{R}}_{23}(0)+\dot{\widehat{R}}_{34}(0)$, when $(i, j, k, l)$ are all $(1, \overline{1})$ on proceeds as in the foregoing sections. For $(i, j, k, l)$, respectively, 2 , the state reduces (due to $2=\overline{2}$ ) to (with $(i, j, k$ ) each $(1, \overline{1}))$

$$
\begin{aligned}
& \left(1+\epsilon_{1}\right)|2\rangle\left(|j\rangle+\epsilon_{2}|\bar{j}\rangle\right)\left(|k\rangle+\epsilon_{2}|\bar{k}\rangle\right)\left(|l\rangle+\epsilon_{4}|\bar{l}\rangle\right), \\
& \left(1+\epsilon_{2}\right)\left(|i\rangle+\epsilon_{1}|\bar{i}\rangle\right)|2\rangle\left(|k\rangle+\epsilon_{2}|\bar{k}\rangle\right)\left(|l\rangle+\epsilon_{4}|\bar{l}\rangle\right), \\
& \left(1+\epsilon_{3}\right)\left(|i\rangle+\epsilon_{1}|\bar{i}\rangle\right)\left(|j\rangle+\epsilon_{2}|\bar{j}\rangle\right)|2\rangle\left(|l\rangle+\epsilon_{4}|\bar{l}\rangle\right), \\
& \left(1+\epsilon_{4}\right)\left(|i\rangle+\epsilon_{1}|\bar{i}\rangle\right)\left(|j\rangle+\epsilon_{2}|\bar{j}\rangle\right)\left(|k\rangle+\epsilon_{3}|\bar{k}\rangle\right)|2\rangle .
\end{aligned}
$$

The factors ( 2 for $\epsilon=1$ for each case) can be ignored. The eigenvalues are in order

$$
\begin{aligned}
& m_{21}^{\left(\epsilon_{2}\right)}+m_{11}^{\left(\epsilon_{2} \epsilon_{3}\right)}+m_{11}^{\left(\epsilon_{3} \epsilon_{4}\right)} \\
& m_{11}^{\left(\epsilon_{1}\right)}+m_{21}^{\left(\epsilon_{2}\right)}+m_{11}^{\left(\epsilon_{3} \epsilon_{4}\right)}, \\
& m_{11}^{\left(\epsilon_{1} \epsilon_{2}\right)}+m_{12}^{\left(\epsilon_{2}\right)}+m_{21}^{\left(\epsilon_{3}\right)}, \\
& m_{11}^{\left(\epsilon_{1} \epsilon_{2}\right)}+m_{11}^{\left(\epsilon_{2} \epsilon_{3}\right)}+m_{12}^{\left(\epsilon_{3}\right)} .
\end{aligned}
$$


For multiple Index 2, one can have

$$
\begin{aligned}
(i, j, k, l)= & (2,2, k, l),(2, j, 2, l),(2, j, k, 2), \\
& (i, 2,2, l),(i, 2, k, 2),(i, j, 2,2),(2,2,2, l), \\
& (2,2, k, 2),(2, j, 2,2),(i, 2,2,2), \\
& (2,2,2,2) .
\end{aligned}
$$

The corresponding states, eigenvalues, and multiplicities are easily written down factorizing out more and more $\epsilon$ 's. For example, for $(i, j, k, l)=(2,2, k, l)$, one has

$$
\left(1+\epsilon_{1}\right)\left(1+\epsilon_{2}\right)|2\rangle|2\rangle\left(|k\rangle+\epsilon_{3}|\bar{k}\rangle\right)\left(|l\rangle+\epsilon_{4}|\bar{l}\rangle\right)
$$

with eigenvalue

$$
m_{22}+m_{21}^{\left(\epsilon_{3}\right)}+m_{11}^{\left(\epsilon_{3} \epsilon_{4}\right)}
$$

One ends with

$$
H^{(3)}|2222\rangle=3 m_{22}|2222\rangle .
$$

4.1.3. $r>3$. The situation is analogous for all $r$, with ever increasing possibilities and subcases as $r$ increases. We do not intend to track down such proliferations systematically. Let us, however, briefly indicate some essential features. Now, $H^{(r)}=\dot{\widehat{R}}_{12}(0)+\cdots+\dot{\widehat{R}}_{r, r+1}(0)$. One, correspondingly, starts with base states

$$
\left(\left|i_{1}\right\rangle+\epsilon_{1}\left|\overline{i_{1}}\right\rangle\right)\left(\left|i_{2}\right\rangle+\epsilon_{2}\left|\overline{i_{2}}\right\rangle\right) \cdots\left(\left|i_{r+1}\right\rangle+\epsilon_{r+1}\left|\overline{i_{r+1}}\right\rangle\right) .
$$

When each $(i, \bar{i})$ is $(1, \overline{1}),|2\rangle$ being absent, one proceeds as in Section 2 . The multiplicity of $|2\rangle$ can now be (when present)

$$
(0,1,2, \ldots, r+1) \text {. }
$$

The number of possibilities (generalizing results as (92)) for the subsets is, respectively,

$$
\left(\begin{array}{c}
r+1 \\
0
\end{array}\right),\left(\begin{array}{c}
r+1 \\
1
\end{array}\right),\left(\begin{array}{c}
r+1 \\
2
\end{array}\right), \ldots,\left(\begin{array}{c}
r+1 \\
r
\end{array}\right),\left(\begin{array}{c}
r+1 \\
r+1
\end{array}\right) .
$$

One ends with

$$
H^{(r)}|22 \cdots 2\rangle=r m_{22}|22 \cdots 2\rangle .
$$

4.2. $C B C$. The Index $2(\overline{2})$ again plays a special role concerning $\mathrm{CBC}$ for $N=3$. For states involving only $(1, \overline{1})$, one proceeds exactly as for $N=2$ (Section 2 ). But $|2\rangle$ now leads to additional possibilities as shown below.

\subsection{1. $r=1$. Apart from}

$$
H^{(1)}(|11\rangle \pm|\overline{11}\rangle)=m_{11}^{( \pm)}(|11\rangle \pm|\overline{11}\rangle),
$$

one now has also

$$
H^{(1)}|22\rangle=m_{22}|22\rangle \text {. }
$$

4.2.2. $r=2$. Apart from periodic states (corresponding to (39))

$$
\begin{aligned}
& H^{(2)}(|111\rangle-|\overline{1} 1 \overline{1}\rangle)=\left(m_{11}^{(+)}+m_{11}^{(-)}\right)(|111\rangle-|\overline{1} 1 \overline{1}\rangle), \\
& H^{(2)}(|\overline{111}\rangle-|1 \overline{1} 1\rangle)=\left(m_{11}^{(+)}+m_{11}^{(-)}\right)(|\overline{111}\rangle-|1 \overline{1} 1\rangle),
\end{aligned}
$$

one now has also

$$
\begin{gathered}
H^{(2)}(|212\rangle+\epsilon|2 \overline{1} 2\rangle)=\left(m_{21}^{(\epsilon)}+m_{12}^{(\epsilon)}\right)(|212\rangle+\epsilon|2 \overline{1} 2\rangle), \\
H^{(2)}|222\rangle=2 m_{22}|222\rangle .
\end{gathered}
$$

4.2.3. $r>2$. In (43), the sum on the left eliminates the (NP) part of (42). For states of the class (as compared to (42))

$$
|2\rangle\left(\left|i_{2}\right\rangle+\epsilon_{2}\left|\overline{i_{2}}\right\rangle\right) \cdots\left(\left|i_{r}\right\rangle+\epsilon_{r}\left|\overline{i_{r}}\right\rangle\right)|2\rangle,
$$

a sum like (43) is no longer necessary. One has a periodic state with

$$
\begin{aligned}
H^{(r)} & \left(|2\rangle\left(\left|i_{2}\right\rangle+\epsilon_{2}\left|\overline{i_{2}}\right\rangle\right) \cdots\left(\left|i_{r}\right\rangle+\epsilon_{r}\left|\overline{i_{r}}\right\rangle\right)|2\rangle\right) \\
= & \left(m_{2 i_{2}}^{\left(\epsilon_{2}\right)}+m_{i_{2} i_{3}}^{\left(\epsilon_{2} \epsilon_{3}\right)}+\cdots+m_{i_{r-1} i_{r}}^{\left(\epsilon_{r-1} \epsilon_{r}\right)}+m_{i_{r} 2}^{\left(\epsilon_{r}\right)}\right) \\
& \times\left(|2\rangle\left(\left|i_{2}\right\rangle+\epsilon_{2}\left|\overline{i_{2}}\right\rangle\right) \cdots\left(\left|i_{r}\right\rangle+\epsilon_{r}\left|\overline{i_{r}}\right\rangle\right)|2\rangle\right)
\end{aligned}
$$

if $\left(i_{2}, \ldots, i_{r}\right)$ are each $(1, \overline{1})$. If one or more of them are (2), the corresponding $\epsilon$ 's factor out as $(1+\epsilon)$ giving (for $\epsilon=+1$ ) a set of periodic states spanning a space of corresponding lesser dimension. Since a sum like (43) is not here necessarily, a problem like that analyzed for $N=4$ (see (67)-(74)) does not arise.

5. $(N, r)$

We are now in a position to consider the situation for $N$ and for any chain length $r$. In fact, after our detailed study of the cases $N=2,3,4$ in the previous sections for all $r$ values $(r=$ $2,3, \ldots)$, no really new technic is necessary for $N>4$. As the domains of the indices labeling the states spanning the base space increase as

$$
\begin{array}{r}
i=(1, \overline{1}),(2, \overline{2}), \ldots,(n, \bar{n}) \quad(\text { for } \quad N=2 n), \\
i=(1, \overline{1}),(2, \overline{2}), \ldots,(n-1, \overline{n-1}), \quad(n=\bar{n}), \\
(\text { for } N=2 n-1),
\end{array}
$$

the dimensions of the base space increase as $N^{r+1}$ displaying explicitly all eigenvalues, and each one with its specific multiplicity becomes prohibitive, not due to subtle new features, but due to the sheer length of enumeration necessary. We do not intend to meet this aspect head on. But we claim that the essential problems have been solved in the preceding sections. let us consider examples. For $N=6$,

$$
i=(1, \overline{1}),(2, \overline{2}),(3, \overline{3}) \text {. }
$$


In constructing periodic states (for $\mathrm{CBC)}, i_{1}$ in (73) can now be not only $(1,1,2,2)$ but also $(3,3)$. But the constraints $(73)$ remain formally the same. For $N=5$,

$$
i=(1, \overline{1}),(2, \overline{2}),(3=\overline{3}) \text {. }
$$

Here, features arising for $N=4$,

$$
i=(1, \overline{1}),(2, \overline{2}) \text {, }
$$

and or $N=3$,

$$
i=(1, \overline{1}),(2=\overline{2})
$$

will coexist. But they can be analyzed systematically combining the formalisms developed for $N=4$ (Section 3 ) and $N=3$ (Section 4). Similar statements hold for higher $N$.

\section{Higher Order "Hamiltonians" (Conserved Quantities)}

In Section 4 of [5], higher order Hamiltonians were briefly discussed (see also Section 1.5 of [6]). For simplicity, we consider here only even dimensions $(N=2,4, \ldots)$. (For odd dimensions one obtains corresponding results by starting from (4.20) of [5].) For even $N$,

$$
\left.\frac{d^{l}}{d \theta^{l}} \widehat{R}(\theta)\right|_{\theta=0}=\sum_{i, j, \epsilon}\left(m_{i j}^{(\epsilon)}\right)^{l}\left(P_{i j}^{(\epsilon)}+P_{i \bar{j}}^{(\epsilon)}\right) .
$$

This crucial result is to be implemented in

$$
H_{l}=\left.\frac{d^{l}}{d \theta^{l}} \log \mathrm{T}^{(\epsilon)}(\theta)\right|_{\theta=0}
$$

Here, $H_{1}$ is our previous $H$. Now, we set

$$
H=H_{1}+c_{1} H_{2}+c_{3} H_{3}+\cdots
$$

For $l=2$, for example, one obtains for a $r$-chain (as a generalization of (11))

$$
\begin{aligned}
\left.\frac{d^{2}}{d \theta^{2}}\left(\widehat{R}_{12}(\theta) \otimes \widehat{R}_{23}(\theta) \otimes \cdots \otimes \widehat{R}_{r, r+1}(\theta)\right)\right|_{\theta=0} \\
=\ddot{\widehat{R}}_{12}(0) \otimes I \otimes \cdots \otimes I+\dot{\widehat{R}}_{12}(0) \otimes \dot{\widehat{R}}_{23}(0) \otimes \cdots \otimes I \\
\quad+\cdots+I \otimes I \otimes \cdots \otimes \ddot{\widehat{R}}_{r, r+1}(0)
\end{aligned}
$$

Implementing the basic result (110), one can collect together the coefficients in a particular simple form.

Let us start with a simple example, which, however, clearly indicates how to perform generalization. For $(r=2, l=2)$ with $a=(i, \bar{i})$ and so on, as compared to (23)-(29), one obtains

$$
\begin{aligned}
H_{2}\left(|a\rangle+\epsilon_{1}|\bar{a}\rangle\right)\left(|b\rangle+\epsilon_{2}|\bar{b}\rangle\right)\left(|c\rangle+\epsilon_{3}|\bar{c}\rangle\right) \\
=\left(\left(m_{a b}^{\left(\epsilon_{1} \epsilon_{2}\right)}\right)^{2}+m_{a b}^{\left(\epsilon_{1} \epsilon_{2}\right)} m_{b c}^{\left(\epsilon_{2} \epsilon_{3}\right)}\right. \\
\left.\quad+m_{b c}^{\left(\epsilon_{2} \epsilon_{3}\right)} m_{a b}^{\left(\epsilon_{1} \epsilon_{2}\right)}+\left(m_{b c}^{\left(\epsilon_{2} \epsilon_{3}\right)}\right)^{2}\right) \\
\quad \times\left(|a\rangle+\epsilon_{1}|\bar{a}\rangle\right)\left(|b\rangle+\epsilon_{2}|\bar{b}\rangle\right)\left(|c\rangle+\epsilon_{3}|\bar{c}\rangle\right) \\
=\left(m_{a b}^{\left(\epsilon_{1} \epsilon_{2}\right)}+m_{b c}^{\left(\epsilon_{2} \epsilon_{3}\right)}\right)^{2} \\
\quad \times\left(|a\rangle+\epsilon_{1}|\bar{a}\rangle\right)\left(|b\rangle+\epsilon_{2}|\bar{b}\rangle\right)\left(|c\rangle+\epsilon_{3}|\bar{c}\rangle\right) .
\end{aligned}
$$

The general result, derived iteratively, is with (as in (32)), for $r$-chain,

$$
\begin{aligned}
V= & \left(\left|a_{1}\right\rangle+\epsilon_{1}\left|\bar{a}_{1}\right\rangle\right)\left(\left|a_{2}\right\rangle+\epsilon_{2}\left|\bar{a}_{2}\right\rangle\right) \cdots \\
& \left(\left|a_{r+1}\right\rangle+\epsilon_{r+1}\left|\bar{a}_{r+1}\right\rangle\right), \\
H_{l} V= & \left(m_{a_{1} a_{2}}^{\left(\epsilon_{1} \epsilon_{2}\right)}+m_{a_{2} a_{3}}^{\left(\epsilon_{2} \epsilon_{3}\right)}+\cdots+m_{a_{r} a_{r+1}}^{\left(\epsilon_{r} \epsilon_{r+1}\right)}\right)^{2} V .
\end{aligned}
$$

This involves a systematic use of (111) and a stepwise generalization of (114).

We now briefly study the higher order Hamiltonians that modify the transition matrix elements. We again concentrate on a particular simple example. With in (110) $c_{l}=0(l>2)$ and $r=2$ in (115), (116),

$$
\begin{aligned}
|a b c\rangle= & \frac{1}{2^{3}}((|a\rangle+|\bar{a}\rangle)+(|a\rangle-|\bar{a}\rangle)) \\
& \times((|b\rangle+|\bar{b}\rangle)+(|b\rangle-|\bar{b}\rangle)) \\
& \times((|c\rangle+|\bar{c}\rangle)+(|c\rangle-|\bar{c}\rangle))
\end{aligned}
$$

expressing $|a b c\rangle$ as a linear combination of basis states appearing in (115). Next, we apply (116) on the product $(|a\rangle+$ $\left.\epsilon_{1}|\bar{a}\rangle\right)\left(|b\rangle+\epsilon_{2}|\bar{b}\rangle\right)\left(|c\rangle+\epsilon_{3}|\bar{c}\rangle\right)$ for all $\left(\epsilon_{1}, \epsilon_{2}, \epsilon_{3}\right)$ and collect together terms with coefficients $\left(m_{a b}^{(\epsilon)}+m_{b c}^{\left(\epsilon^{\prime}\right)}\right)$. We obtain (for terms with nonzero coefficients)

$$
\begin{aligned}
4 H_{1}|a b c\rangle & \\
= & \left(m_{a b}^{(+)}+m_{b c}^{(+)}\right)(|a b c\rangle+|a \overline{b c}\rangle+|\bar{a} b \bar{c}\rangle+|\bar{a} \bar{b} c\rangle) \\
& +\left(m_{a b}^{(+)}+m_{b c}^{(-)}\right)(|a b c\rangle-|a \overline{b c}\rangle-|\bar{a} b \bar{c}\rangle+|\bar{a} \bar{b} c\rangle) \\
& +\left(m_{a b}^{(-)}+m_{b c}^{(+)}\right)(|a b c\rangle+|a \overline{b c}\rangle-|\bar{a} b \bar{c}\rangle-|\bar{a} \bar{b} c\rangle) \\
& +\left(m_{a b}^{(-)}+m_{b c}^{(-)}\right)(|a b c\rangle-|a \overline{b c}\rangle+|\bar{a} b \bar{c}\rangle-|\bar{a} \bar{b} c\rangle)
\end{aligned}
$$

or

$$
\begin{aligned}
H_{1}|a b c\rangle=\frac{1}{2}( & \left(m_{a b}^{(+)}+m_{a b}^{(-)}+m_{b c}^{(+)}+m_{b c}^{(-)}\right)|a b c\rangle \\
& +\left(m_{a b}^{(+)}+m_{a b}^{(-)}\right)|\bar{a} \bar{b} c\rangle \\
& \left.+\left(m_{b c}^{(+)}-m_{b c}^{(-)}\right)|a \bar{b} \bar{c}\rangle\right) .
\end{aligned}
$$


Note that, for $H_{1}$, the coefficient of $|\bar{a} \bar{b} \bar{c}\rangle$ is zero on the right. We now compute the action of $\mathrm{H}_{2}$ on $|a b c\rangle$. Using the decomposition (117) and (116) with $l=2$, one obtains on carefully grouping terms the following result:

$$
H_{2}|a b c\rangle=f_{0}|a b c\rangle+f_{1}|a \bar{b} \bar{c}\rangle+f_{2}|\bar{a} b \bar{c}\rangle+f_{3}|\bar{a} \bar{b} c\rangle
$$

with

$$
\begin{gathered}
4 f_{0}=\left(\left(m_{a b}^{(+)}+m_{b c}^{(+)}\right)^{2}+\left(m_{a b}^{(+)}+m_{b c}^{(-)}\right)^{2}\right. \\
\left.+\left(m_{a b}^{(-)}+m_{b c}^{(+)}\right)^{2}+\left(m_{a b}^{(-)}+m_{b c}^{(-)}\right)^{2}\right), \\
2 f_{1}=\left(m_{a b}^{(+)}+m_{a b}^{(-)}+m_{b c}^{(+)}+m_{b c}^{(-)}\right)\left(m_{b c}^{(+)}-m_{b c}^{(-)}\right), \\
2 f_{2}=\left(m_{a b}^{(+)}-m_{a b}^{(-)}\right)\left(m_{b c}^{(+)}-m_{b c}^{(-)}\right), \\
2 f_{3}=\left(m_{a b}^{(+)}-m_{a b}^{(-)}\right)\left(m_{a b}^{(+)}+m_{a b}^{(-)}+m_{b c}^{(+)}+m_{b c}^{(-)}\right) .
\end{gathered}
$$

Note that, for $l=2$, the coefficient of $|\bar{a} b \bar{c}\rangle$ is no longer zero (as for $l=1$ ) for general values of parameters.

Decomposing $\left|a_{1} a_{2} \cdots a_{r} a_{r+1}\right\rangle$ as in (117) to give factors $\left(\left|a_{k}\right\rangle+\epsilon_{k}\left|\bar{a}_{k}\right\rangle\right)(k=1,2, \ldots, r)$ which appear in eigenfunctions (115), implementing the action of $H_{l}$ (from (116)) and then again collecting together coefficients of states generalizing the right hand members of (120), one obtains transition matrix elements for all $(r, l)$. We will not try to present such results explicitly. The foregoing ones clearly indicate the successive steps. However, some general features are worth noting. When $r$ increases, the action of (112) on basis states induced of simultaneous flips between different closely or well separately sites. The corresponding transition matrix elements can be obtained systematically for the full action of (112) if so desired. Short and long range correlations can thus be extracted and explicitly formulated.

\section{Discussions}

The central feature of our class of braid matrices and associated Hamiltonians is the number of free parameters (of the order of $N^{2}$ ) coexisting with simple symmetries permitting systematic, explicit construction of eigenstates and eigenvalues for all dimensions and chain lengths $(N, r)$.

There exists a rich class of multidimensional, multiparameter Yang-Baxter (and hence braid) matrices [7, 8], where progress has been made in the construction of eigenstates of transfer matrices [9]. (See the discussion added in [5].) For this class, however, the parameters enter via multiple rapidities (in contrast to our single $\theta$ ), making the situation basically different. For our case the symmetries leading to complete, explicit solutions restrict the properties of the models. But the solutions show how one can pass from one sector to another by varying the relative magnitudes of the parameters.

The simplest case, $N=2$ becomes trivial for $m_{11}^{(+)}=m_{11}^{(-)}$. For $m_{11}^{(+)}>m_{11}^{(-)}$, the highest and the lowest eigenvalues (for chain length $r$ ) are, respectively, $r m_{11}^{(+)}$and $r m_{11}^{(-)}$. The situation is reversed for $m_{11}^{(+)}<m_{11}^{(-)}$. The relative spacing of the levels depend on $m_{11}^{(+)}-m_{11}^{(-)}$. It is also clearly seen how the highest and lowest levels $r m_{11}^{( \pm)}$are excluded for closed chains (as noted below (41)). For $N>2$, the number of parameters, increasing as $N^{2}$, leads to elaborating possibilities for classifying accessible sectors. For $N=4$, one already has 8 parameters and the general expression for the eigenvalues for chain length $r$ (from (61)) is $\left(m_{i_{1} i_{2}}^{\left(\epsilon_{1} \epsilon_{2}\right)}+m_{i_{2} i_{3}}^{\left(\epsilon_{2} \epsilon_{3}\right)}+\right.$ $\left.\cdots+m_{i_{r} i_{r+1}}^{\left(\epsilon_{r} \epsilon_{r+1}\right)}\right)$, where each $i$ can be $(1, \overline{1}, 2, \overline{2})$ and each $\epsilon$ can be $(+,-)$ independently. This and its direct generalization for all $N$ display the possibilities concerning the number of sectors and crossovers according to $m$ 's chosen. We cannot propose physical significances corresponding to such multisector patterns. One may contrast it, however, with the domains of the single parameter of the 6-vertex model and the associated (antiferromagnetic, critical, and ferromagnetic) regimes [10, 11]. We have presented a thorough study of eigenstates and eigenvalues. But other directions remain to be explored. We intend to study elsewhere correlations in our models. It would be interesting to compare the situation with those encountered for famous familiar cases [12].

It would be interesting to study higher order conserved magnitudes obtained through higher order $\theta$-derivatives of the braid matrix [6]. We intend to generalize in another direction, the nested sequence of projectors (2) that can be generalized by including parameters. A $4 \times 4$ example can be found in the paper where nested sequences were introduced [13]. It appeared in the context of $\mathscr{U}_{q}\left(\widehat{s l_{2}}\right)$ (Section 5, [13]). We aim to present a systematic generalization of higher dimensions and corresponding Baxterized braid matrices.

Finally, we briefly point out that the Hamiltonians studied here can be carried over without change for a class of unitary braid matrices presented in the context of entanglement [14]. It was pointed out $[3,14]$ that for all $m$ 's imaginary $\left(m_{j k}^{(\epsilon)} \rightarrow\right.$ i $m_{j k}^{(\epsilon)}$, with $m$ 's real on the right) $\widehat{R}(\theta)$ of (4) becomes unitary. A further overall factor $-\mathrm{i}$ gives the same $\dot{\widehat{R}}(0)$ as for real $m$ 's. Hence, the result, in [14], another class of unitary $\widehat{R}(\theta)$, was constructed. Namely,

$$
\widehat{R}^{ \pm 1}(\theta)=\frac{1}{\sqrt{1+\tanh ^{2} \theta}}(I \otimes I \pm \tanh \theta K \otimes J)
$$

where

$$
J=\sum_{i=1}^{n}\left((-1)^{\bar{i}}(\bar{i} \bar{i})+(-1)^{i}(\bar{i} i)\right), \quad K=\sum_{i=1}^{n}((\bar{i} \bar{i})+(\bar{i} i)) .
$$

Now,

$$
\dot{\widehat{R}}(0)=K \otimes J
$$

contains no parameters. The Hamiltonians can be studied quite simply. 


\section{References}

[1] A. Chakrabarti, "A nested sequence of projectors and corresponding braid matrices $\hat{R}(\theta)$ : (1) Odd dimensions," Journal of Mathematical Physics, vol. 46, no. 6, Article ID 063508, 2005.

[2] B. Abdesselam and A. Chakrabarti, "Nested sequence of projectors. II. Multiparameter multistate statistical models, Hamiltonians, S-matrices," Journal of Mathematical Physics, vol. 47, no. 5, Article ID 053508, 2006.

[3] B. Abdesselam, A. Chakrabarti, V. K. Dobrev, and S. G. Mihov, "Higher dimensional multiparameter unitary and nonunitary braid matrices: even dimensions," Journal of Mathematical Physics, vol. 48, no. 10, Article ID 103505, 2007.

[4] B. Abdesselam and A. Chakrabarti, "A new eight vertex model and higher dimensional, multiparameter generalizations," Journal of Mathematical Physics, vol. 49, no. 5, Article ID 053301, 2008.

[5] B. Abdesselam and A. Chakrabarti, "Multiparameter statistical models from $N^{2} \times N^{2}$ braid matrices: explicit eigenvalues of transfer matrices $\mathrm{T}^{(r)}$, spin chains, factorizable scatterings for all N," Advances in Mathematical Physics. In press, http://arxiv.org/abs/0806.2371.

[6] H. Saleur and J. B. Zuber, "Integrable lattice models and quantum groups," in Proceedings of the 1990 Trieste Spring School on String Theory and Quantum Gravity, 1990.

[7] J. H. H. Perk and C. L. Schultz, "New families of commuting transfer matrices in $q$-state vertex models," Physics Letters A, vol. 84, no. 8, pp. 407-410, 1981.

[8] J. H. H. Perk and H. Au-Yang, "Yang-Baxter equations," in Encyclopedia of Mathematics and Physics, vol. 5, pp. 465-473, Elsevier, Oxford, UK, 2006.

[9] C. L. Schultz, "Eigenvectors of the multi-component generalization of the six-vertex model," Physica A, vol. 122, no. 1-2, pp. 71-88, 1983.

[10] R. J. Baxter, Exactly Solved Models in Statistical Mechanics, Academic Press, New York, NY, USA, 1982.

[11] H. J. De Vega, "Yang-Baxter algebras, integrable theories and quantum groups," International Journal of Modern Physics A, vol. 4, p. 2371, 1989.

[12] V. E. Korepin, G. Izergin, and N. M. Bogoliubov, Quantum Inverse Scattering Method, Correlation Functions and Algebraic Bethe Ansatz, Cambridge University Press, Cambridge, UK, 1993.

[13] A. Chakrabarti, "Canonical factorization and diagonalization of Baxterized braid matrices: explicit constructions and applications," Journal of Mathematical Physics, vol. 44, no. 11, pp. 53205349, 2003.

[14] B. Abdesselam, A. Chakrabarti, V. K. Dobrev, and S. G. Mihov, "Higher dimensional unitary braid matrices: construction, associated structures, and entanglements," Journal of Mathematical Physics, vol. 48, no. 5, Article ID 053508, 2007. 


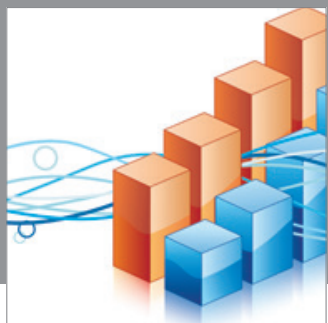

Advances in

Operations Research

mansans

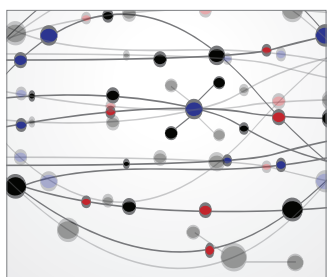

The Scientific World Journal
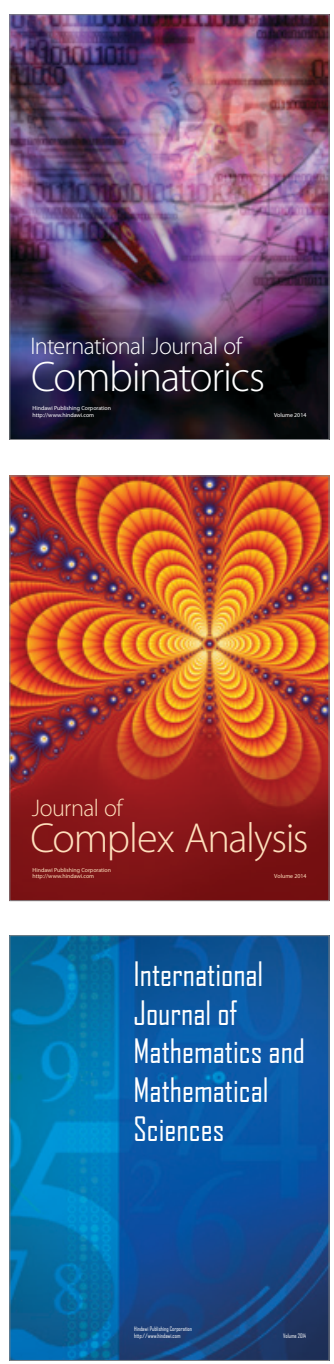
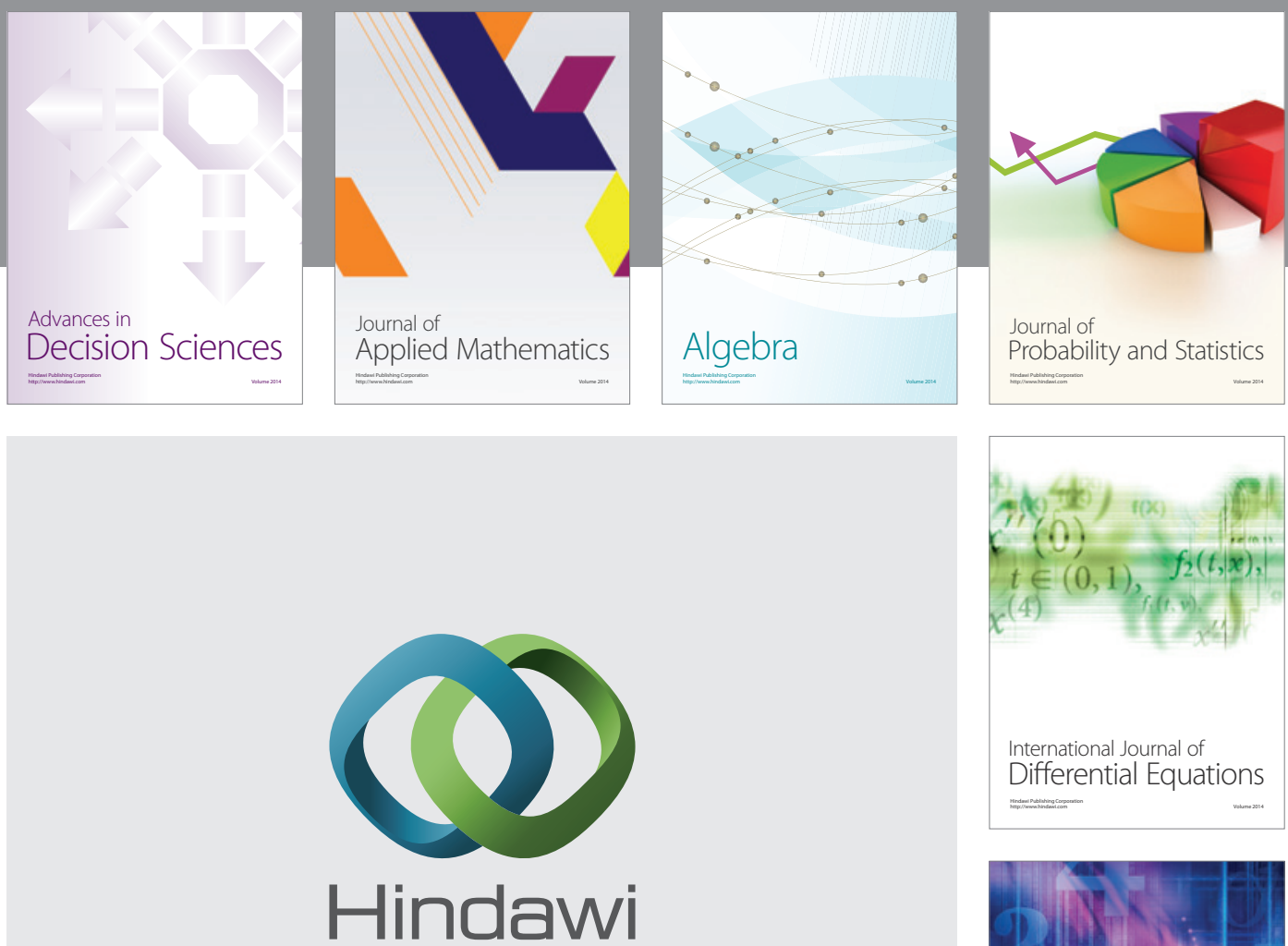

Submit your manuscripts at http://www.hindawi.com
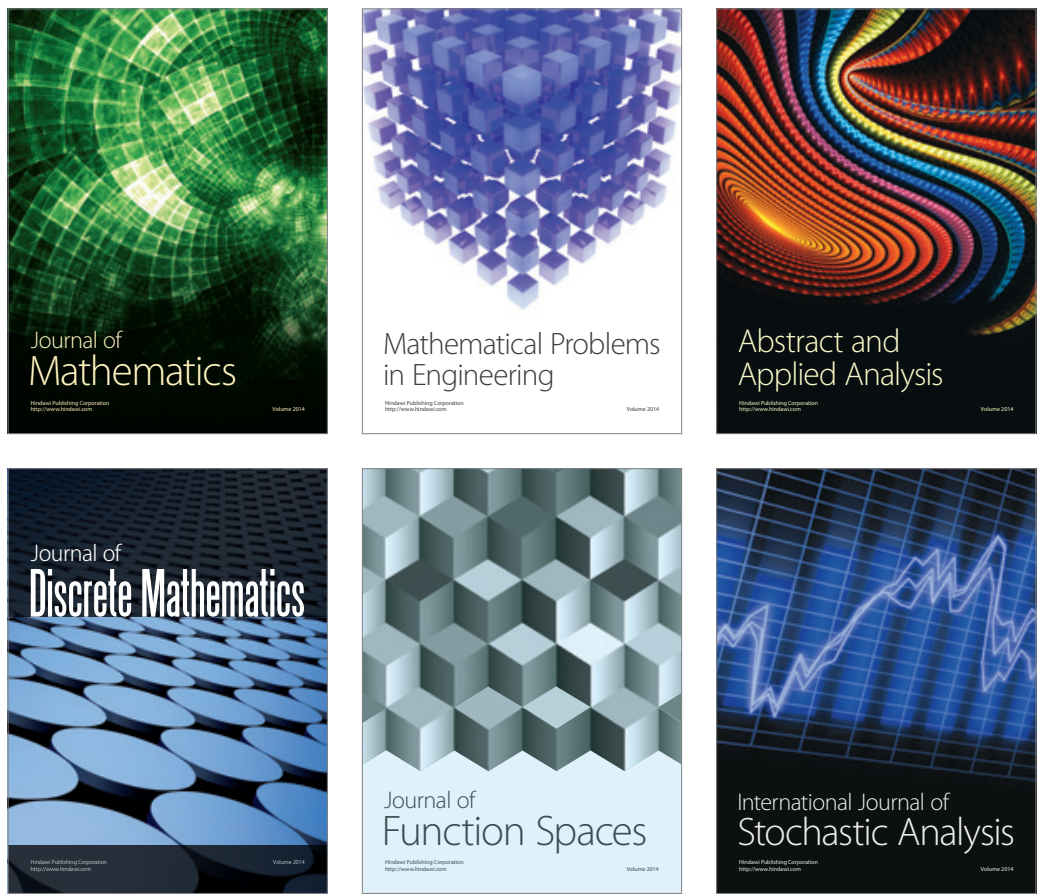

Journal of

Function Spaces

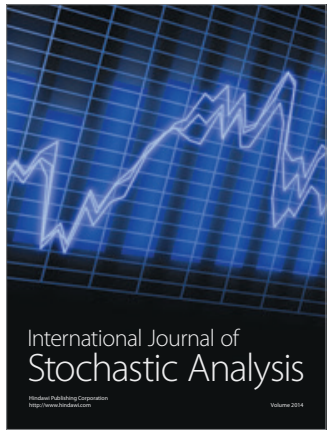

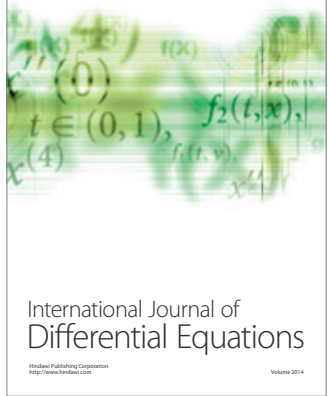
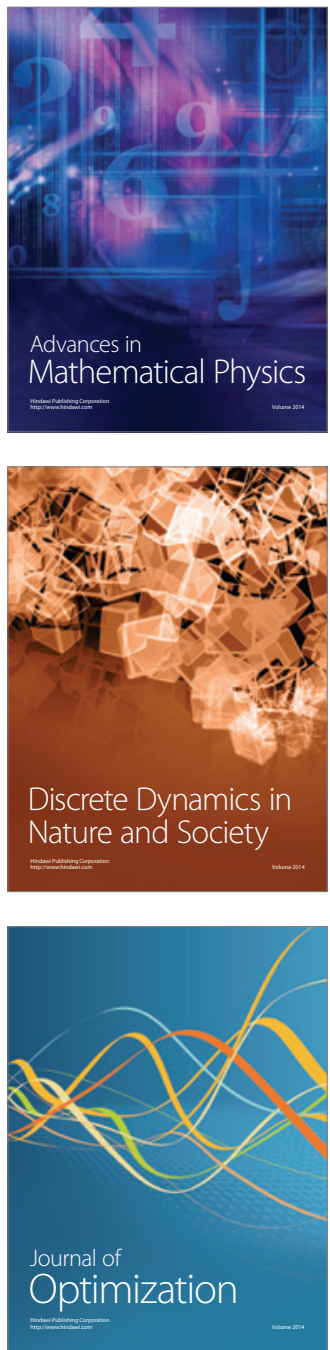